\title{
A DEEP LEARNING-BASED APPROACH FOR DETECTION OF DEMENTIA FROM BRAIN MRI
}

\author{
Reshma L ${ }^{1}$, Sai Priya Nalluri ${ }^{2}$, Priya R Sankpal ${ }^{3}$ \\ ${ }^{1}$ Student, BNM Institute of Technology-Bengaluru, Karnataka, India \\ ${ }^{2}$ Student, BNM Institute of Technology-Bengaluru, Karnataka, India \\ ${ }^{3}$ Professor, BNM Institute of Technology-Bengaluru, Karnataka, India \\ 1reshmalswamy@gmail.com,2²saipriya200@gmail.com,_3riyarsankpal@gmail.com
}

\begin{abstract}
In this paper, a user-friendly system has been developed which will provide the result of medical analysis of digital images like magnetization resonance of image scan of brain for detection and classification of dementia. The small structural differences in the brain can slowly and gradually become a major disease like dementia. Progression of dementia can be slowed when identified early. Hence, this paper aims at developing a robust system for classification and identifying dementia at the earliest. The method used in this paper for initial disclosure and diagnosis of dementia is deep learning since it can give important results in a shorter period of time. Deep Learning methods such as K-means clustering, Pattern Recognition and Multi-class Support Vector Machine (SVM) have been used to classify different stages of dementia. The goal of this study is to provide a user interface for deep learning-based dementia classification using brain magnetic resonance imaging data. The results show that the created method has an accuracy of $96 \%$ and may be utilized to detect people who have dementia or are in the early stages of dementia.
\end{abstract}

Keywords: Dementia, MRI, K-means clustering, Pattern Recognition, Multi-class SVM.

\section{INTRODUCTION}

Dementia is a collection of conditions determined by deterioration of at least two brain functions, such as memory loss and judgement. Dementia is characterized by a group of conditions and as a result, it is not a particular illness. It is an all-inclusive term for a slew of symptoms linked to a decrease in memory or other cognitive abilities severe enough to impair a person's ability to carry out everyday tasks [1]. The various causes of dementia are as follows:

- Alzheimer's Disease: $60-80 \%$ of the total cases.

- Lewy body Dementia: $5-10 \%$ of the total cases.

- Vascular Dementia: 5-10\% of the total cases.

- Frontotemporal Dementia: 5-10\% of the total cases.

- Others: Parkinson's Disease, Huntington's Disease account for $<5 \%$ of the cases.

However, there are a variety of other disorders that might be mistaken for dementia symptoms, including those that are curable but are often overlooked, such as vitamin insufficiency and thyroid issues.

Dementia can be broadly classified into four stages:

- Mild cognitive impairment

- Mild dementia

- Moderate dementia

- Severe Dementia 
These stages are explained in Table 1.

Table 1. Characteristics and Symptoms of various stages of Dementia.

\begin{tabular}{|c|c|c|c|}
\hline Sl. No. & Stages of Dementia & Characteristics & Symptoms \\
\hline 1. & Stage-I & $\begin{array}{l}\text { Mild cognitive } \\
\text { impairment }\end{array}$ & $\begin{array}{l}\text { Subtle decline in } \\
\text { thinking }\end{array}$ \\
\hline 2. & Stage-II & Mild dementia & $\begin{array}{c}\text { Memory changes, } \\
\text { confusion }\end{array}$ \\
\hline 3. & Stage-III & Moderate dementia & $\begin{array}{c}\text { Inability to eat, } \\
\text { bathe or dress } \\
\text { without help }\end{array}$ \\
\hline 4. & Stage-IV & Severe Dementia & $\begin{array}{l}\text { Loss of ability to } \\
\text { communicate and } \\
\text { recognize loved } \\
\text { ones }\end{array}$ \\
\hline
\end{tabular}

These stages are detected using Magnetic Resonance Imaging (MRI) which is a diagnostic imaging technique that presently offers the most accurate non-invasive way of imaging the spinal cord, brain, and other areas of the human body. It is the most commonly used imaging method to help confirm the diagnosis of dementia and to survey the course of the disease [3,4]. Visualization and understanding about the underline pathology of the disease has been made easier due to MRI scans.

The number of individuals living with dementia is expected to rise quickly as average life expectancy rises. By 2050 it is predicted that 131.5 million people will be affected by dementia as shown in Figure 1 [4,5].

In light of this information, a strong system to track demented people and their illness development is required, reducing the load on those liable for their care.

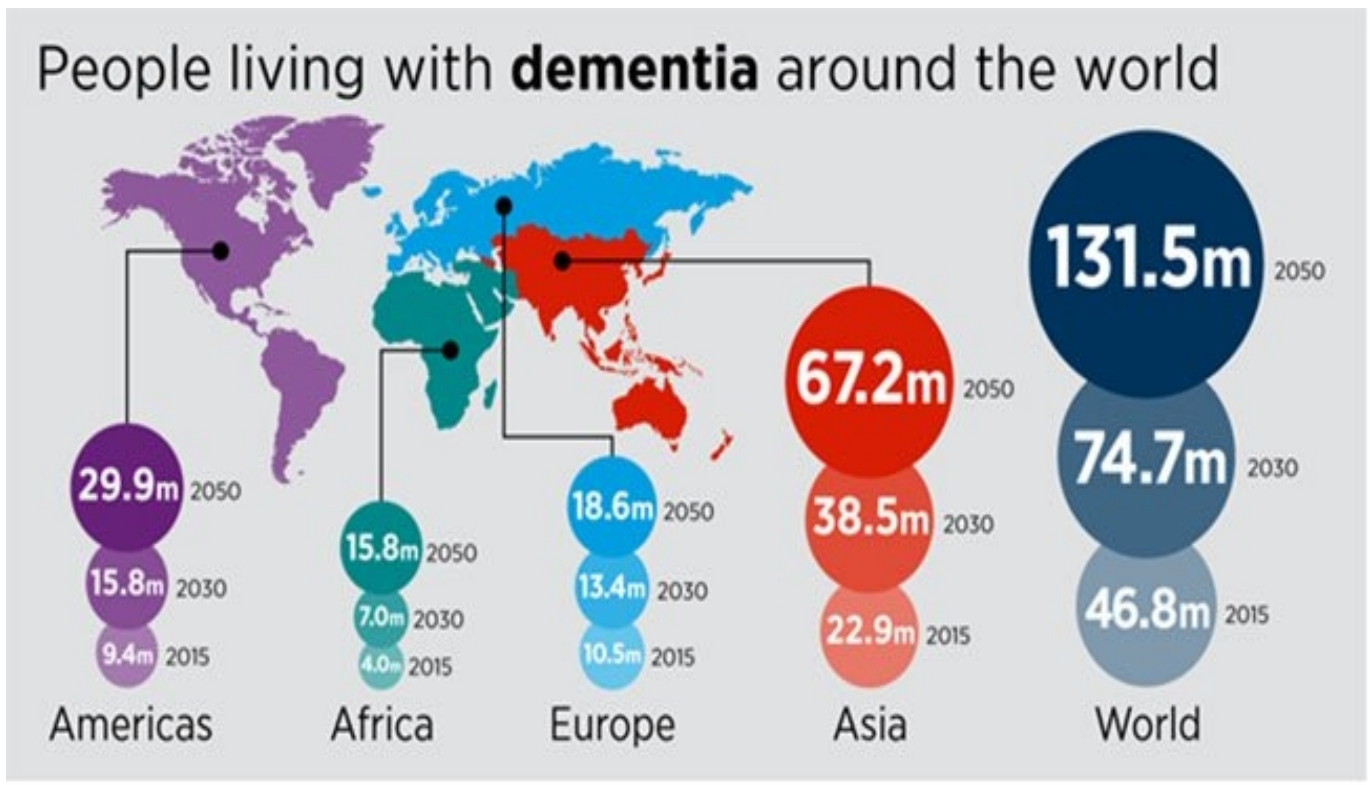

Figure 1. Estimation of the number of people that will be affected by Dementia. 


\section{RELATED WORKS}

For Alzheimer's disease detection, Shrikant Patro and Prof. Nisha V M employed Bicubic Interpolation and Watershed Segmentation [6]. They suggested an image processing technique for processing coronal, axial, and sagittal plane magnetic resonance imaging (MRI) of the brain. In a brain MRI, image segmentation is utilized to identify the afflicted region. The regions of the brain that have been identified using MRI include hippocampus and brain volume It is determined who has Alzheimer's disease, who has mild cognitive impairment, and who belongs to the healthy cohort.

L. Jaba Sheela and Dr. V. Shanthi later employed the CBA algorithm to achieve the same goal [7]. Harlick proposes five textural characteristics based on the MRI images that define the disease. An updated CBA technique is utilized to identify the pictures as Normal or Abnormal, which is supported by the rule set established during the training phase. The tests were conducted on the OASIS dataset, and the results demonstrate that the proposed system classifies with $98 \%$ accuracy, showing the application of association rules in classification.

For the detection of Alzheimer's disease, Rigel Mahmood and Bishad Ghimire employed supervised learning [8]. They developed a new approach for better classification of Alzheimer's disease based on mathematical and image processing approaches. The most frequent method these days is to analyse MRI scans using diffeomorphism principles to create a mapping from one MRI to another.

Hiroki Fuse, Kota Oishi, Norihide Maikusa, and Tadanori Fukami investigated the application of Shape Analysis of MRI Images using Contour extraction and Feature extraction by P-type Fourier descriptor to identify Alzheimer's disease. The lateral ventricle, including the septum lucidum, was examined using a P-type Fourier Descriptor as shape information in this paper. They used a Support Vector Machine to do classification utilising a mixture of multiple descriptors as features (SVM). The classification accuracy was $87.5 \%$, which was superior than the accuracy achieved using the volume ratio to intracranial volume $(81.5 \%)$, which is frequently used to evaluate morphological change [9].

P. Padilla, M. López, J. M. Górriz, J. Ramrez, D. Salas-González, and I. lvarez created an NMFSVM based CAD tool that used Intensity Normalization and Fisher Discriminant Ratio for feature selection and achieved an accuracy of $85-91 \%$. The functional brain scans are examined and divided using a computer-aided scripting language. A single photon emission computed tomography (SPECT) database and positron emission tomography (PET) images were used for this purpose, both of which comprised data for both Alzheimer's Disease patients and healthy subjects as a benchmark [10]. The Fisher discriminant ratio (FDR) and nonnegative matrix factorization are used to evaluate these databases.

However, these papers failed to classify the various stages of dementia. In this paper, a deep learning-based network is used to train the different types of dementia features along with the label data set. This trained network is used to classify the test case images into different stages and classes. 


\section{METHODOLOGY}

Multiple image processing techniques were used to pre-process the MRI images of the brain such as contrast enhancement, filtering, segmentation, morphological processing etc. These images are then fed into pattern recognition neural network where they are trained [11]. The block diagram of the method used in the study is shown in Figure 2. These steps are explained in the successive sections.

In this study, 104 T2-weighted MRI images of various stages of dementia in .jpg format have been considered. The size of the images range between $250-800 \mathrm{kB}$.

\subsection{Image Acquisition}

The database images used contain structural MRI images taken by a means of a medical camera. The database images are collected from OASIS- 2 and the size of the images is not standard.

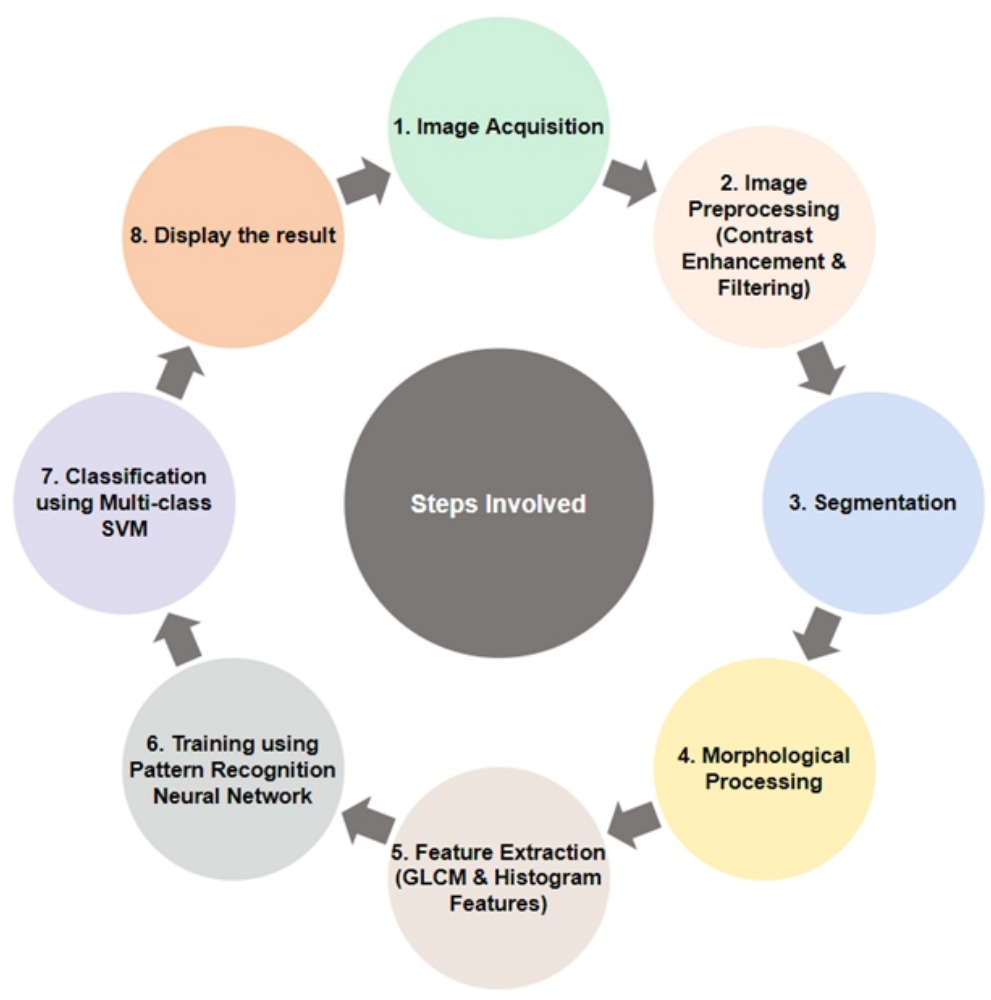

Figure 2. Block Diagram of the proposed system.

\subsection{Image Pre-processing}

The use of a computer to manipulate digital images is known as digital processing. Because raw data from imaging sensors contains faults, it must go through several stages of processing to eliminate these flaws and ensure information originality. Contrast enhancement is a technique for making visual characteristics stand out more clearly by making good use of the display's colors or output device. Later noise is removed using median filter [11,12]. The steps involved are shown in Figure 3. 


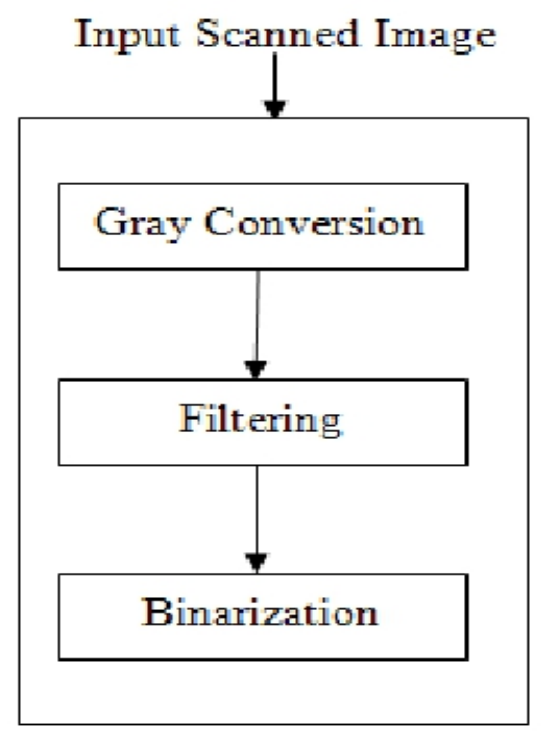

Figure 3. Pre-processing Block Diagram.

\subsection{Segmentation}

These images are segmented after they have been pre-processed. Segmentation is the process of dividing an image into distinct zones using pixels with the same axis. To be relevant and useful for image analysis and evaluation, the reasoning must have a strong connection to the exhibited item or feature of interest. From low-level image processing to high-level image representation in terms of features, objects, and scenes, meaningful segmentation is the first step in transforming a grayscale image into one or more other images. The quality of segmentation determines the effectiveness of image analysis, yet precise image partitioning is a tough challenge to tackle [13]. Segmentation outcomes that are commonly obtained for an MRI image are shown in Figure 4. In this study we have used K-means Clustering which will be discussed in further sections.

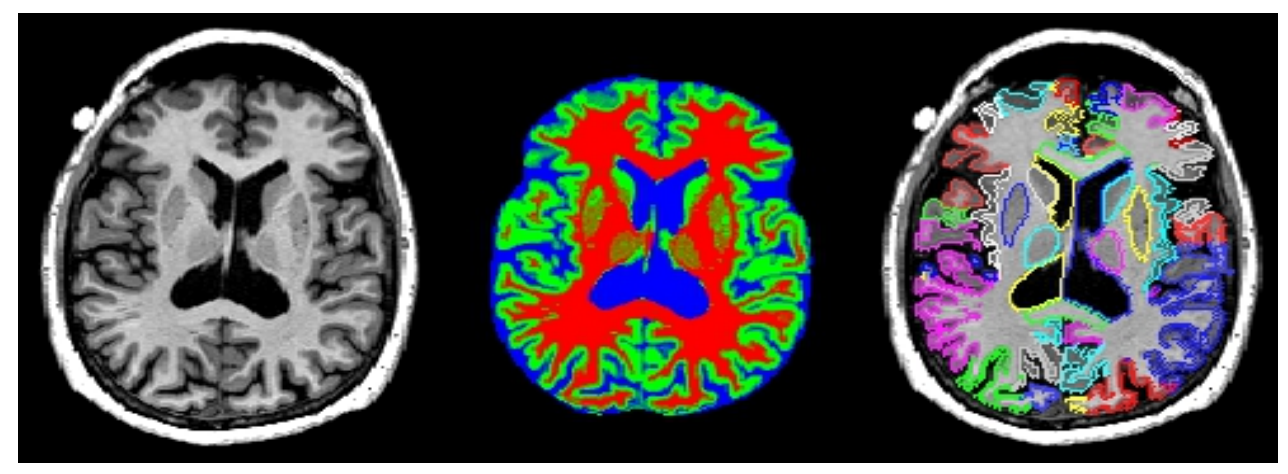

Figure 4. Segmentation of an MRI.

\subsection{Morphological Operations}

Morphological operation aims at removing imperfections caused due to noise and texture caused by distortion in the binary regions of the image. Hence a non-linear set of operations associated to the morphology or shape of features is used [13]. Various types of morphological operations are illustrated in Figure 5. 


\section{a. Original}

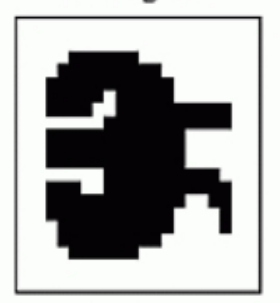

b. Erosion

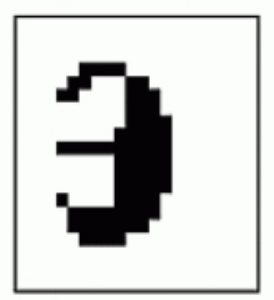

c. Dilation

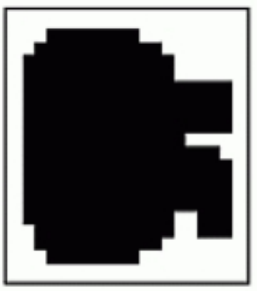

d. Opening

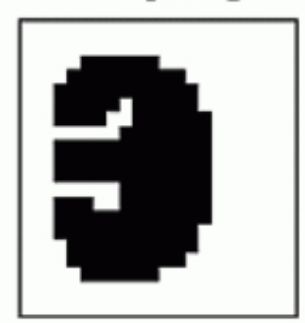

e. Closing

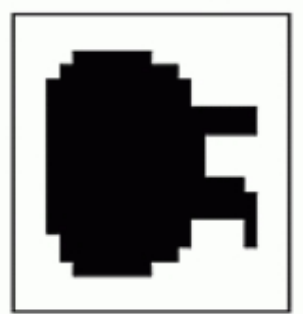

Figure 5. Morphological operations.

\subsection{Feature Extraction}

Feature extraction is a form of dimensionality reduction that condenses a huge amount of raw data into a smaller group that can be processed. The new set of features should then be able to highlight the old set's array of data. In this approach, a condensed version of the original feature can be made by combining the original set of images [14]. The common types of features extracted are shown in Figure 6.

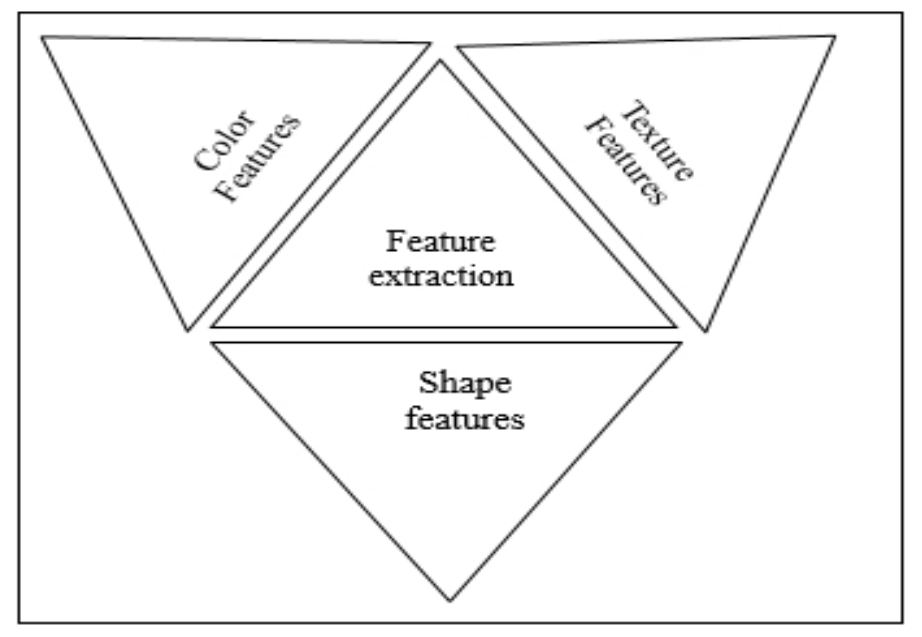

Figure 6. Various Features.

\subsection{Pattern Recognition Neural Network}

Data categorization and grouping are aided by neural networks. They help in grouping unlabeled data based on similarities among the exampled inputs when they have a labelled data set to train on, and they classify data when they have a labelled data set to train on. Deep neural networks are parts of larger machine learning systems that include classification, reinforcement learning, and regression techniques. Neural networks can extract characteristics that are then input into clustering and classification algorithms. During this study we have used Pattern recognition neural network. These networks are used for training of data based on knowledge already gained from statistical information [15]. Their applications include speech recognition, speaker identification and automatic medical diagnosis. The steps involved in mapping features using a pattern recognition network is shown in Figure 7. 


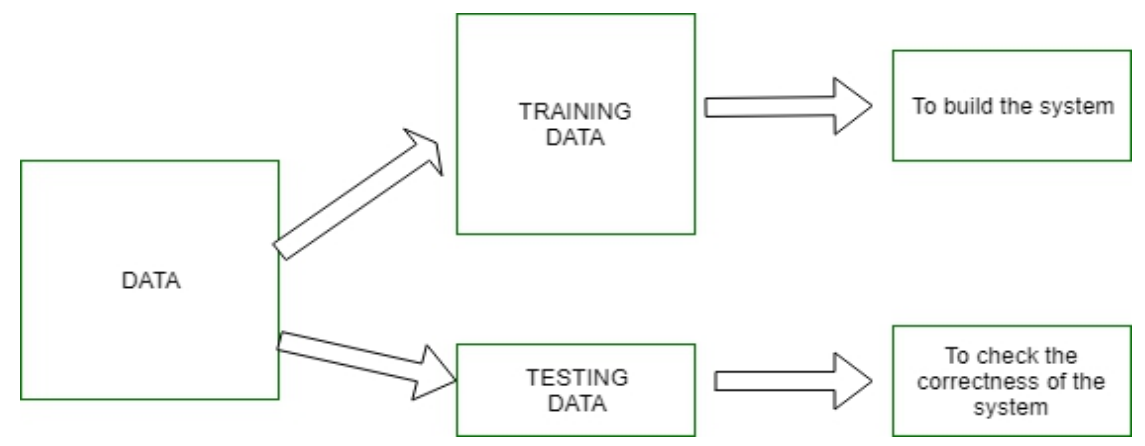

Figure 7. Block diagram of pattern recognition neural network.

\subsection{Multi-class SVM}

The formal definition of a support vector machine (SVM) is a discriminative classifier with a separating hyper plane. In other words, given labelled training data, the algorithm generates an optimum hyper plane that categorizes new samples (supervised learning).

Multi class SVM works on the principal of one-to-rest. Here the classifier can use m-SVMs. Each SVM would predict membership in one of the ' $m$ ' classes [16]. Classified results obtained using an SVM is shown in Figure 8.

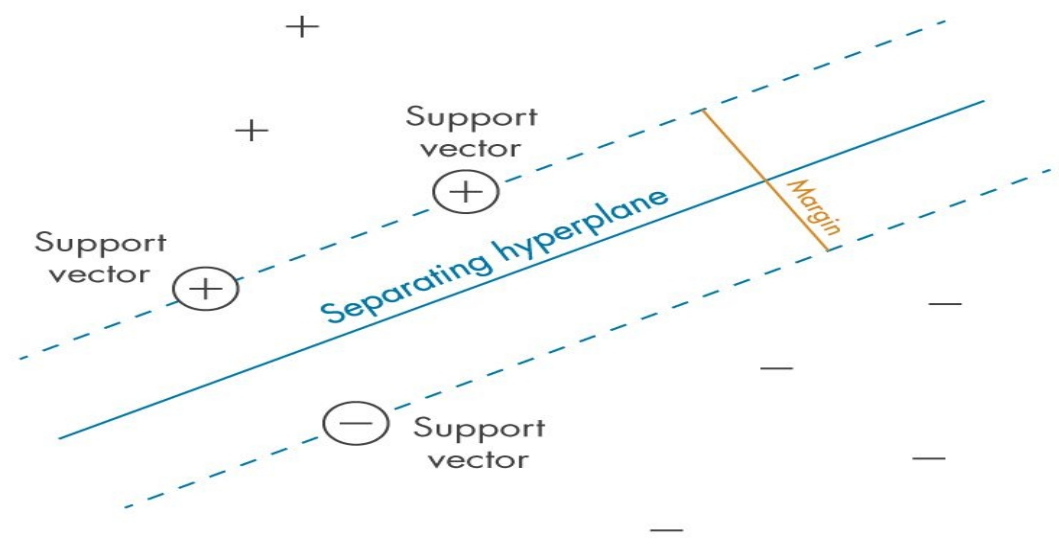

Figure 8. Classification using SVM.

\section{EXPERIMENTAL RESULTS}

The implemented GUI contains a total of 9 push buttons each corresponding to the set to be executed. MATLAB's graphical user interface design environment (guide) is used to create the GUI.

\subsection{Push button 1: Load Image}

When the load image button is clicked, MATLAB will direct us to a folder in our local system where the data set for our study is stored. The selected image is then uploaded in the GUI.

\subsection{Push button 2: Contrast enhancement}

Before performing contrast enhancement, the chosen image is first converted into a gray scale image. Then, contrast enhancement is performed using adaptive histogram equalization as it provides better clarity. This method works on small areas of an image rather than the entire image. Contrast of each pixel is enhanced hence the features of the entire image stands out. 


\subsection{Push button 3: Filtering}

Processes implemented along with filtering include smoothing, sharping, and edge enhancement. In this study median filtering has been performed using medfilt2 which is a 2-D filter.

\subsection{Push button 4: Segmentation}

Initially a colorspace function is used to convert a color image to LAB colorspace. Lab colorspace stands for $\mathrm{L}$ for lightness and $\mathrm{a}$ and $\mathrm{b}$ for the color dimension. It is a colorspace with 3 -axis color system. The obtained output is segmented using K-means clustering algorithm.

\section{K-means clustering}

Clustering is one of the most common exploratory data analysis technique used to get an intuition about the structure of the data. It can be defined as the task of identifying subgroups in the data such that data points in the same subgroup (cluster) are very similar while data points in different cluster are very different. The decision of the similarity measure used is application-specific. K-means is one of the simplest unsupervised learning algorithms that solves the clustering problem [13]. The procedure follows a simple and easy way to classify a given data set through a certain number of clusters (k) fixed apriori.

\section{Algorithm of K-means Clustering}

- Determine the values of ' $\mathrm{k}$ '.

- Initialize the centroid.

- Measure the distance.

- Assign to the nearest cluster. If there is no convergence go back to step 3

- If there is convergence measure the variance. Repeat step 2-5 until you get the lowest sum of variance.

- Clustering results are obtained.

Clustering results obtained after segmentation are shown in Figure 9.

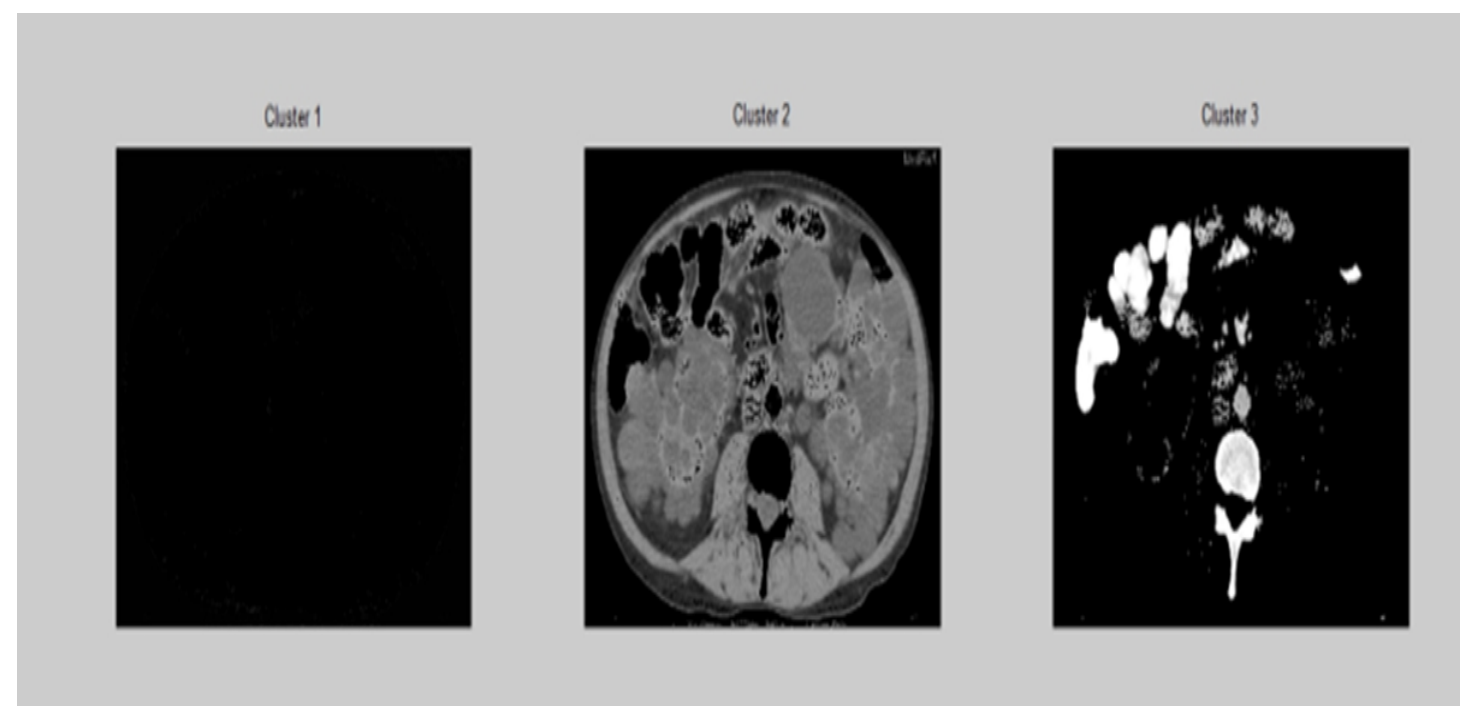

Figure 9. Segmentation using K-means algorithm.

\subsection{Push button 5: Morphological operation}

Firstly, light structures which are connected to the image border are suppressed. Dilation is performed next. It adds pixels to the boundary of objects in an image. 


\subsection{Push button 6: Feature Extraction}

Firstly, a GLCM matrix is created. Then the following properties are obtained.

- Contrast: the intensity contrast between a pixel and its neighbor over the entire image.

- Correlation: It is the statistical measure of how closely a pixel is connected to its neighbors throughout the full image.

- Energy: summation of squared elements in the GLCM.

- Homogeneity: refers to how near the distribution of elements in the GLCM is to the diagonal of the GLCM.

- Entropy: Entropy is a randomness measure that takes low values for smooth images.

- Mean: It is the average value.

- Standard Deviation: This is extracted using std2 i.e. $B=\operatorname{std} 2(\mathrm{~A})$ computes the standard deviation of the values in A.

- RMS: It stands for Root mean squared value. For vectors, $\operatorname{rms}(\mathrm{X})$ is the root mean squared value in $\mathrm{X}$.

- Variance: It is extracted using var. For vectors, $\mathrm{Y}=\operatorname{var}(\mathrm{X})$ returns the variance of the values in $\mathrm{X}$.

- Kurtosis: $\mathrm{K}=$ kurtosis $(\mathrm{X})$ returns the sample kurtosis of the values in $\mathrm{X}$.

- Skewness: $\mathrm{S}=$ skewness $(\mathrm{X})$ returns the sample skewness of the values in $\mathrm{X}$.

- IDM: It stands for Inverse Difference Movement. Inverse difference moment is the measure of local homogeneity.

The extracted features are stored in a .mat file for further steps as shown in Figure 10.

\begin{tabular}{|c|c|c|c|c|c|c|c|c|c|c|c|}
\hline \multirow{2}{*}{\multicolumn{12}{|c|}{$\begin{array}{l}\text { feat_disease } X \\
\mathbb{\boxplus} 1 \times 13 \text { double }\end{array}$}} \\
\hline & & & & & & & & & & & \\
\hline & 1 & 2 & 3 & 4 & 5 & 6 & 7 & 8 & 9 & 10 & 11 \\
\hline 1 & 0.6091 & 0.93309 & 0.0947 & 0.8073 & 86.9241 & 67,5264 & 6.3352 & 13.4297 & $3.5002 \mathrm{ee}+03$ & 1.0000 & 2.7450 \\
\hline \multicolumn{12}{|l|}{2} \\
\hline \multicolumn{12}{|l|}{3} \\
\hline 4 & & & & & & & & & & & \\
\hline
\end{tabular}

Figure 10. Feature extraction table.

\subsection{Push button 7: Training}

In the recent years, Magnetic Resonance Texture Analysis has been picking up pace to determine the level of damage in neuroimaging data based on GLCM and histogram features. This is where pattern recognition network comes into the picture.

The extracted GLCM features from the previous step are trained for classification using a simple feed-forward pattern recognition neural network [16]. The following steps are followed in training this model:

- Create a Pattern Recognition Network using MATLAB in-built function patternet [16].

- The hidden layer is taken as 10 .

- The next process is to set up data division for testing, validation, and training.

- Train the network: This is accomplished using the [NET,TR] $=\operatorname{train}(\mathrm{NET}, \mathrm{X}, \mathrm{T})$ function, which takes a network NET, input data X, and target data T and returns the network as well as a training record TR after training. This is shown in the Figure 11.

- The network is then tested using perform(NET,T,Y,EW), which receives a network, targets $\mathrm{T}$ and outputs $\mathrm{Y}$, and optionally error weights $\mathrm{EW}$, and returns performance using the network's default performance function. This is shown in the Figure 12.

- Finally, the accuracy of the model is calculated. 


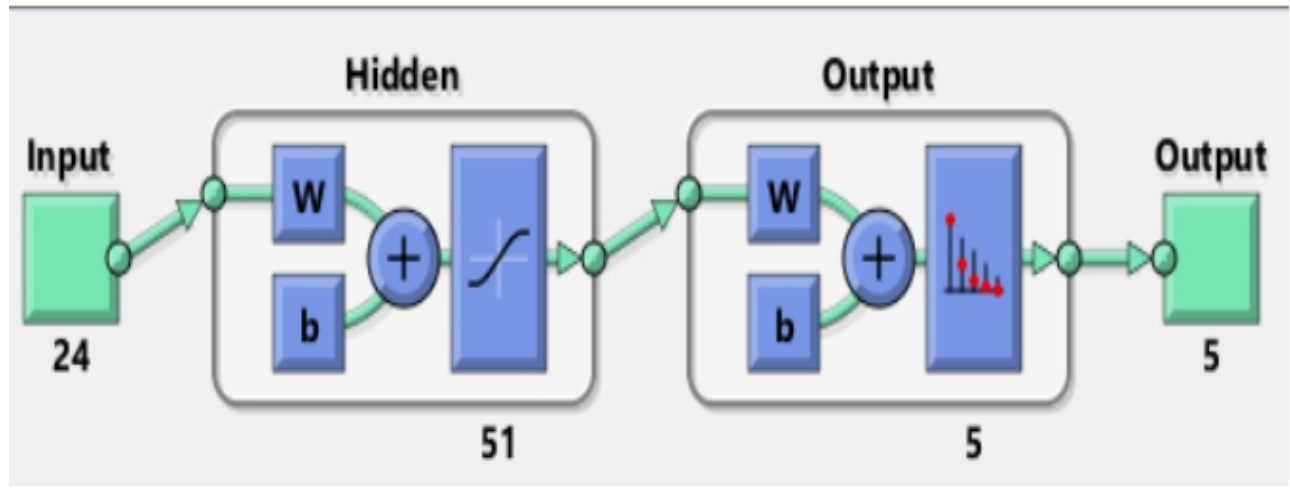

Figure 11. Pattern recognition network.

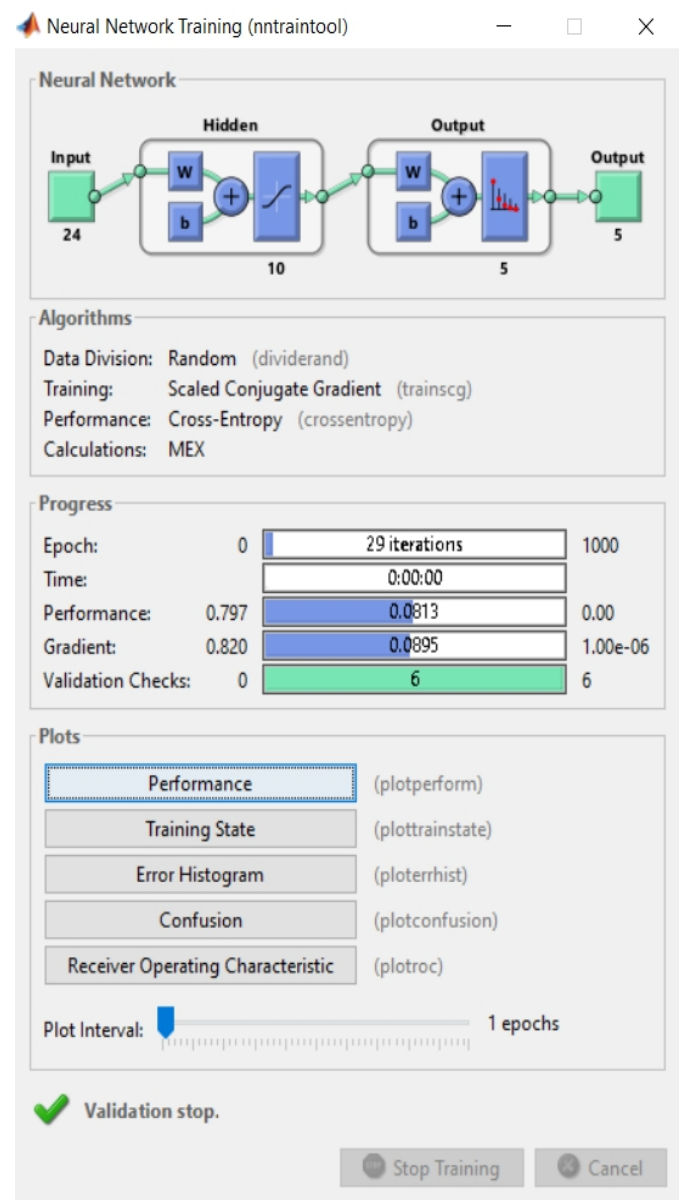

Figure 12. Neural network training.

\subsection{Push button 8: Classification}

In this study we want to classify the level of dementia into four classes i.e. Stage-I (Mild cognitive impairment), Stage-II (mild dementia), Stage-III (moderate dementia) and Stage-IV (severe dementia). For this to happen we first need to teach the classifier on what basis the levels are supposed to be classified. The image is classified using the SVM after comparison of features obtained from the selected image and the pre extracted features which are labeled using pattern recognition network [17]. In more technical terms, an SVM produces a hyperplane or a family of hyperplanes for classification in a high or infinite-dimensional space. 


\section{Steps: SVM}

- Import the data set.

- Explore the data and pre-process them.

- Split the data into attributes and label.

- Train the SVM algorithm and make predictions.

- Evaluate the results.

The classification of various stages of dementia is shown in Figure 13.

\begin{tabular}{|c|c|c|c|c|c|c|c|c|c|c|c|}
\hline & 1 & 2 & 3 & 4 & 5 & 6 & 7 & 8 & 9 & 10 & 11 \\
\hline 1[ & 0.5490 & 0.9209 & 0.1289 & 0.8186 & 54.7048 & 55.8791 & 5.7009 & 12.9882 & $2.6004 e+03$ & 3.4780 & 0.9698 \\
\hline 2 & 0.3877 & 0.9115 & 0.1238 & 0.8515 & 104.8558 & 47.8520 & 6.1474 & 15.9687 & $2.0296 \mathrm{e}+03$ & 3.9457 & 0.1995 \\
\hline 3 & 0.3544 & 0.9543 & 0.2642 & 0.8785 & 59.0329 & 69.8338 & 4.5726 & 10.2549 & $3.8922 \mathrm{e}+03$ & 1.9317 & 0.6582 \\
\hline 4[ & 1.0279 & 0.8168 & 0.0696 & 0.7465 & 126.1819 & 57.8829 & 6.9289 & 15.9687 & $2.7613 e+03$ & 1.8443 & 0.1875 \\
\hline 5 & 0.4300 & 0.9178 & 0.0856 & 0.8246 & 130.3181 & 50.0537 & 7.0119 & 15.9687 & $1.8571 \mathrm{e}+03$ & 3.0293 & $\cdot 0.1262$ \\
\hline 6 & 0.6972 & 0.9121 & 0.0796 & 0.7900 & 83.2737 & 74.8776 & 6.8395 & 13.8334 & $4.3552 \mathrm{e}+03$ & 1.9342 & 0.4510 \\
\hline 7 & 0.9949 & 0.8642 & 0.0553 & 0.7278 & 92.1676 & 59.4338 & 6.8461 & 14.1843 & $2.3389 e+03$ & 2.3148 & -0.2115 \\
\hline 8 & 0.7328 & 0.9085 & 0.1152 & 0.7869 & 55.9730 & 54.9283 & 5.8666 & 13.1243 & $2.6660 \mathrm{e}+03$ & 3.1950 & 0.8370 \\
\hline 9 & 0.5665 & 0.9274 & 0.1248 & 0.8221 & 75.6449 & 61.8568 & 5.9050 & 12.5899 & $2.7269 \mathrm{e}+03$ & 2.1693 & 0.1977 \\
\hline 10 & 0.5856 & 0.9134 & 0.0802 & 0.8038 & 88.1531 & 61.3807 & 6.6693 & 14,4155 & $2.6839 \mathrm{e}+03$ & 2.8341 & 0.4154 \\
\hline 11 & 0.2605 & 0.9595 & 0.2814 & 0.8996 & 46.1219 & 58.2424 & 4.5033 & 10.3318 & $2.7241 e+03$ & 3.2571 & 1.0257 \\
\hline 12 & 0.6091 & 0.9309 & 0.0947 & 0.8073 & 86.9241 & 67.5264 & 6.3352 & 13.4297 & $3.5902 \mathrm{e}+03$ & 2.7450 & 0.3971 \\
\hline 13 & 0.5684 & 0.9258 & 0.1175 & 0.8143 & 66.5784 & 59.9747 & 5.8354 & 12.6513 & $2.5754 \mathrm{e}+03$ & 2.6681 & 0.4453 \\
\hline 14 & 0.6091 & 0.9309 & 0.0947 & 0.8073 & 86.9241 & 67.5264 & 6.3352 & 13.4297 & $3.5902 \mathrm{e}+03$ & 2.7450 & 0.3971 \\
\hline 15 & 1.1476 & 0.8343 & 0.0527 & 0.7073 & 82.6555 & 53.0166 & 6.5356 & 14.0610 & $1.9277 \mathrm{e}+03$ & 2.9698 & $\cdot 0.1249$ \\
\hline \multicolumn{12}{|c|}{$<$} \\
\hline
\end{tabular}

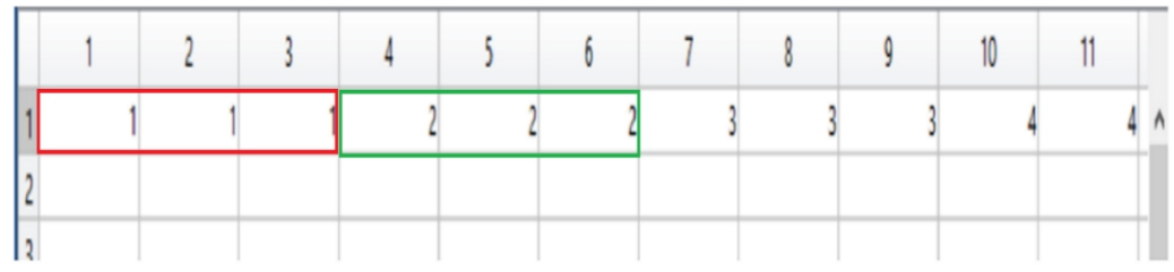

Figure 13. Classification using SVM.

The final outline of GUI is shown in Figure 14. The obtained results are shown in Table 2.

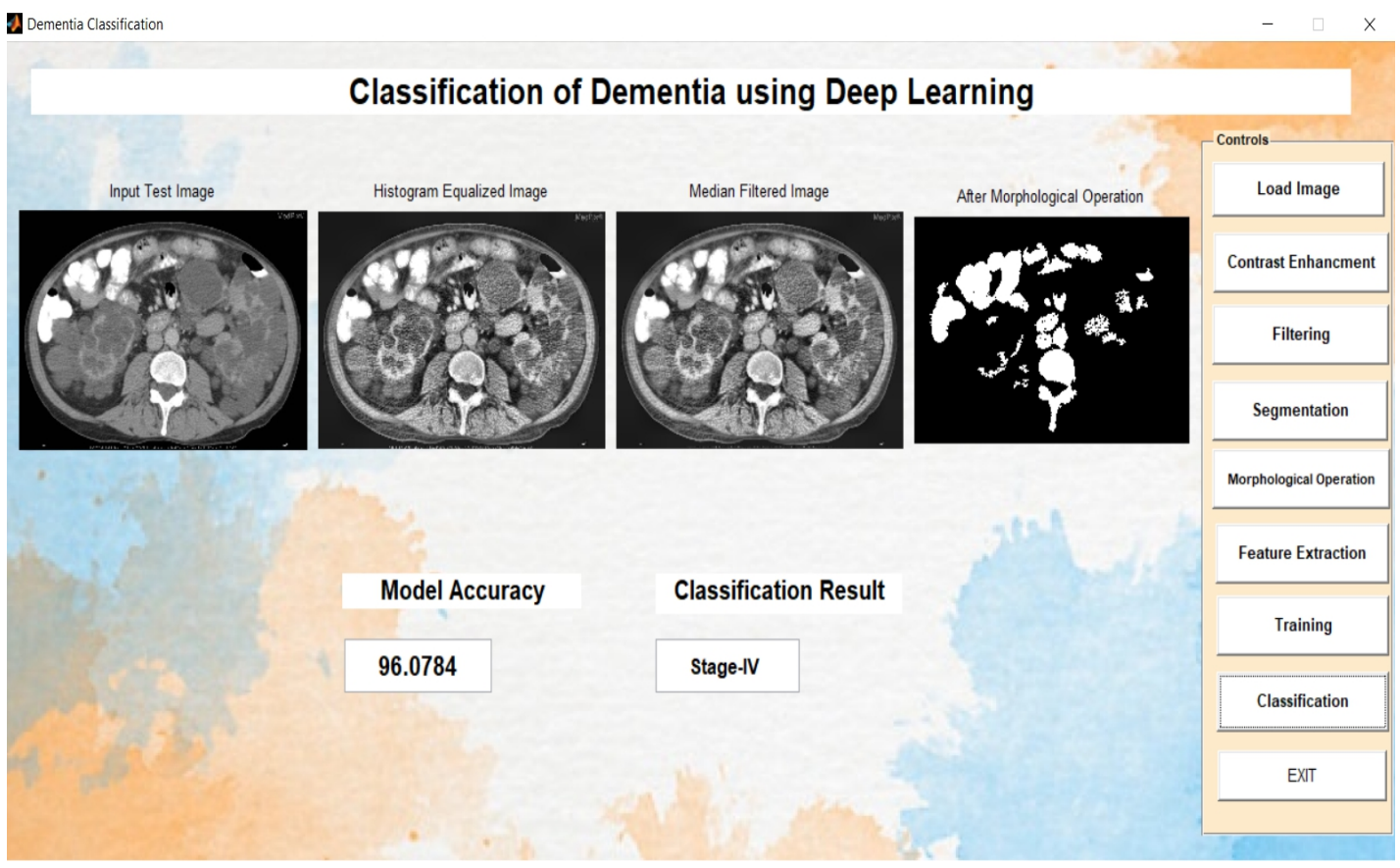

Figure 14. Outline of the GUI. 
Accuracy is calculated using the formula,

Accuracy $=\frac{(\mathrm{TP}+\mathrm{TN})}{(\mathrm{TP}+\mathrm{TN}+\mathrm{FP}+\mathrm{FN})}$

where $\mathrm{TP}=$ True Positives, $\mathrm{TN}=$ True Negatives, FP $=$ False Positives, and FN $=$ False Negatives. These values are obtained from the confusion matrix.

Table 2. Obtained accuracy values

\begin{tabular}{|c|c|}
\hline PercentErrors & Accuracy \\
\hline 0.1 & $96.07 \%$ \\
\hline 0.2 & $88.23 \%$ \\
\hline 0.3 & $86.27 \%$ \\
\hline
\end{tabular}

\section{CONCLUSION}

There is no way to tell if someone has dementia without doing a test. A thorough medical history, physical examination, lab examination, and the typical changes in thought and behavior associated with each type of dementia help doctors diagnose dementia and its type. Doctors can detect whether or not a person has dementia with a high degree of confidence, but determining the kind of dementia is more difficult since the brain alterations of distinct dementias might overlap. Hence in this paper Magnetic resonance texture analysis has been used to differentiate between various stages. The pre-extracted features are trained and then assigned to each class using a pattern recognition neural network and based on this the chosen image is classified into a particular stage using multi class SVM. The model accuracy was found to be about $96 \%$. Majority of the papers which were referred during the study used supervised learning methods such neural networks (CNN, ANN). However, in the study Multi-Class SVM has been used to classify stages of dementia.

To determine whether we can figure out which portions of the brain are damaged by dementia, future enhancements to this research will look at particular regions of the MR image rather than the full image. Accuracy can be improved further by including hybrid optimization techniques with the present mechanisms. Further investigations could also include optimization of classifiers. Investigating the efficiency of the proposed method for a larger data set could be an area of research potential.

\section{Acknowledgments}

We express our heartfelt gratitude to the college management of BNM Institute of Technology, Bengaluru for enabling us to carry out the research work with all the resources and subsequent publication. Also, we express our sincere gratitude to Visvesvaraya Technological Institute (VTU), Belagavi for providing us the research platform.

\section{REFERENCES}

[1] H. M. Tarek Ullah, Zishan Ahmed Onik, Riashat Islam, and Dip Nandi, “Alzheimer's Disease and Dementia Detection from 3D Brain MRI Data Using Deep Convolutional Neural Networks," 2018 3rd International Conference for Convergence in Technology (I2CT), Pune, India, doi: 10.1109/I2CT.2018.8529808.

[2] Doaa Ebrahim, Amr M. T. Ali-Eldin, Hossam E. Moustafa, and Hesham Arafat, "Alzheimer Disease Early Detection Using Convolutional Neural Networks," 2020 15th International Conference on Computer Engineering and Systems (ICCES), Cairo, Egypt, doi: 10.1109/ICCES51560.2020.9334594. 
[3] J A Akhila, Christin Markose and R P Aneesh, "Feature extraction and classification of Dementia with neural network", 2017 International Conference on Intelligent Computing, Instrumentation and Control Technologies (ICICICT), Kerala, India, doi: 10.1109/ICICICT1.2017.8342782.

[4] Maxim Mizotin, Jenny Benois-Pineau, Michèle Allard, and Gwenaelle Catheline, "Featurebased brain MRI retrieval for Alzheimer disease diagnosis", 2012 19th IEEE International Conference on Image Processing, Orlando, FL, USA, doi: 10.1109/ICIP.2012.6467091.

[5] Luis Javier Herrera, Ignacio Rojas, H. Pomares, A. Guillén, O. Valenzuela and O. Baños, "Classification of MRI Images for Alzheimer's Disease Detection”,2013 International Conference on Social Computing, Alexandria, VA, USA, doi:10.1109/SocialCom.2013.127.

[6] Shrikant Patro, Prof. Nisha V M, 2019, Early Detection of Alzheimer's Disease using Image Processing, International Journal of Engineering Research \& Technology (IJERT) Volume 08, Issue 05 (May 2019).

[7] L. J. Sheela and V. Shanthi, "An Image Analysis and Classification Protocol for Characterization of Normal and Abnormal Memory Loss in Aging from Structural MRI," 2009 International Conference on Signal Processing Systems, Singapore, 2009, pp. 639- 643, doi: 10.1109/ICSPS.2009.199.

[8] R. Mahmood and B. Ghimire, "Automatic detection and classification of Alzheimer's Disease from MRI scans using principal component analysis and artificial neural networks," 2013 20th International Conference on Systems, Signals and Image Processing (IWSSIP), Bucharest,2013, pp. 133-137, doi: 10.1109/IWSSIP.2013.6623471.

[9] Hiroki Fuse, Kota Oishi, Norihide Maikusa, and Tadanori Fukami, "Detection of Alzheimer's Disease with Shape Analysis of MRI Images", 2018 Joint 10th International Conference on Soft Computing and Intelligent Systems and 19th International Symposium on Advanced Intelligent Systems, pp. 978-1-5386-2633-7, doi: 10.1109/SCISISIS.2018.00171.

[10] P. Padilla, M. Lopez, J. M. Gorriz, J. Ramirez, D. Salas-Gonzalez, and I. Alvarez, "NMFSVM Based CAD Tool Applied to Functional Brain Images for the Diagnosis of Alzheimer's Disease”, 2012 IEEE Transactions on Medical Imaging, pp. 0278-0062.

[11] A. W. Salehi, P. Baglat, B. B. Sharma, G. Gupta and A. Upadhya, "A CNN Model: Earlier Diagnosis and Classification of Alzheimer Disease using MRI", 2020 International Conference on Smart Electronics and Communication (ICOSEC), Trichy, India, 2020, pp. 156-161, doi: 10.1109/ICOSEC49089.2020.9215402.

[12] Sagar Adatrao and Mayank Mittal, "An analysis of different image preprocessing techniques for determining the centroids of circular marks using hough transform", 2016 2nd International Conference on Frontiers of Signal Processing (ICFSP), Warsaw, Poland, doi:10.1109/ICFSP.2016.7802966.

[13] Xin Zheng, Qinyi Lei, Run Yao, Yifei Gong \& Qian Yin," Image segmentation based on adaptive K-means algorithm", EURASIP Journal on Image and Video Processing volume 2018, doi: 10.1186/s13640-018-0309-3.

[14] P. Mohanaiah, P. Sathyanarayana, and L. GuruKumar, "Image Texture Feature Extraction Using GLCM Approach", 2013 International Journal of Scientific and Research Publications, Volume 3, Issue 5, ISSN 2250-3153.

[15] M. Yu. Mikheev, Yu. S. Gusynina, and T. A. Shornikova, "Building Neural Network for Pattern Recognition”, 2020 International Russian Automation Conference, Sochi, Russia, doi: 10.1109/RusAutoCon49822.2020.9208207. 
[16] Sai Yeshwanth Chaganti, Ipseeta Nanda, Koteswara Rao Pandi, Tavva G.N.R.S.N. Prudhvith, and Niraj Kumar, "Image Classification using SVM and CNN", 2020 International Conference on Computer Science, Engineering and Applications (ICCSEA), Gunupur, India, doi: 10.1109/ICCSEA49143.2020.9132851.

[17] Guang-Bin Huang; Hongming Zhou; Xiaojian Ding; Rui Zhang, "Extreme Learning Machine for Regression and Multiclass Classification", IEEE Transactions on Systems, Man, and Cybernetics, Part B (Cybernetics), doi:10.1109/TSMCB.2011.2168604 